\title{
Prévalence et susceptibilité aux antibiotiques des souches de Salmonella spp. non typhiques isolées de la viande de poulets au Tchad
}

\author{
Hamadou ABBA ${ }^{1,2}$, Marius K. SOMDA ${ }^{3 *}$, Ban-bo Bebanto ANTIPAS ${ }^{2}$, Nicolas BARRO ${ }^{3}$ \\ et Alfred S. TRAORE ${ }^{3}$ \\ ${ }^{I}$ Laboratoire de Biologie de la Faculté des Sciences Exactes et Appliquées de L'Université de N'Djaména, \\ Tchad. \\ ${ }^{2}$ Laboratoire de Biologie de Hôpital de la Mère et Enfant de N'Djaména, Tchad. \\ ${ }^{3}$ Centre de Recherche en Sciences Biologiques, Alimentaires et Nutritionnelles (CRSBAN). Département de \\ Biochimie-Microbiologie (DBM), Unité de Formation et de Recherche en Sciences de la Vie et de la Terre \\ (UFR/SVT), Université Ouagal Pr Joseph KI-ZERBO, 03 BP 7021, Ouagadougou 03, Burkina Faso. \\ *Auteur correspondant ;E-mail: somdasmarius@yahoo.fr
}

\section{RESUME}

L'utilisation non contrôlée d'antibiotiques en élevage conduit à la sélection des germes résistants, à la recrudescence des infections, l'augmentation de la mortalité et la baisse de la productivité en viande de poulet au Tchad. Cette étude a pour but de déterminer la prévalence et la susceptibilité des souches de Salmonella spp. Isolées dans les carcasses de poulets au Tchad aux antibiotiques. Au total 60 échantillons de carcasses de poulets ont été prélevés dans les sites d'élevage et point de vente. Les souches de Salmonella spp. ont été isolées suivant les méthodes standard de microbiologiques et confirmées par la galérie Api 20 E. La sensibilité aux antibiotiques a été déterminée par la méthode de diffusion sur milieu solide. La contamination à Salmonella spp. a été détectée dans $68,33 \%$ des échantillons provenant des sites d'élevages et 34,15\% des points de vente. Les résultats des tests de sensibilité aux antibiotiques ont montré que : 92,68\% des Salmonella isolées étaient résistantes à au moins un antibiotique, $100 \%$ des souches présentaient une résistance à l'ampicilline, érythromycine et à l'imipenème ; $87,80 \%$ à l'acide fusidique et $51,22 \%$ à la tétracycline. Un pourcentage de $34,15 \%$ de multi-résistance des souches a été observé. Cette étude a permis d'évaluer la prévalence et d'apprécier le profil d'antibiorésistance des souches de Salmonella spp. isolées.

(C) 2016 International Formulae Group. All rights reserved.

Mots clés : Poulet de chair, Salmonella spp, prévalence, antibiotique, résistance, Tchad.

\section{Prevalence and susceptibility to antibiotics of strains of non-typhoid Salmonella spp. isolated from chicken meat in Chad}

\begin{abstract}
The non controlled use of antibiotics has conducted raising to the selection of the resistant germs, to the upsurge of the infections, the increase of mortality and the decrease of the productivity to Chad. This study has aimed to determine the prevalence and the susceptibility to the antibiotics of Salmonella spp. strains isolated from carcass of chickens in Chad. Sixty (60) samples of chicken's carcass have been collected to the stock
\end{abstract}


farming and sales site. The strains of Salmonella spp. have been isolated according to microbiological standard methods and confirmed by Kit Api 20 E. The sensitivity to the antibiotics has been determined by the method of diffusion on solid medium. The contamination to Salmonella spp. has been detected in $68.33 \%$ of samples coming from the stock farming and $34.15 \%$ of sales site. The results of the sensitivity test to the antibiotics showed that: $92.68 \%$ of Salmonella spp. isolated were resistant to at least an antibiotic, $100 \%$ of the strains presented a resistance to ampicilline, erythromycine and to the imipeneme; $87.80 \%$ to the acidic and $51.22 \%$ to the tetracycline. A percentage of $34.15 \%$ of multi-resistance of the strains has been observed. This study allowed evaluating prevalence and to appreciate the profile of antibioresistance of Salmonella spp. strains isolated.

(C) 2016 International Formulae Group. All rights reserved.

Keywords: Broiler of frams, Salmonella spp., prevalence, antibiotic, resistance, Chad.

\section{INTRODUCTION}

Les maladies d'origine alimentaire sont une cause importante de morbidité et de mortalité à travers le monde. En effet, White et al. (1997), estiment que les diarrhées tuent 3 millions d'enfants chaque année. Aux EtatsUnis, on estime que 1,4 millions de personnes sont infectées par des salmonelles non typhiques chaque année, avec 15000 hospitalisations et 400 morts, alors qu'en France, le nombre est estimé à 30000 cas de salmonelloses avec entre 92 et 535 morts (Humpreh, 2000 ; Voetsch et al., 2004).

Dans les pays en voie de développement, la fièvre typhoïde fait 600000 morts et les salmonelloses non typhiques sont responsables de diarrhées aiguës. En Afrique subsaharienne, ces dernières liées à la contamination de l'eau et des aliments par les selles, aboutissent au décès de 22 à $45 \%$ des personnes infectées (Kingsley et al., 2012).

Toutefois, pour limiter l'influence négative des pathologies majeures rencontrées dans les élevages dont les salmonelloses, les éleveurs ont recours à l'utilisation abusive de médicaments vétérinaires, en particulier les antibiotiques (Biagui, 2002; Molla, 2003; Gaudel, 2013). D'autres auteurs tels: Alambedji et al. (2004), ont révélé la présence de résidus d'antibiotiques dans la chair de poulets dans environ $10 \%$ des élevages enquêtés. Les risques potentiels liés à la présence des résidus d'antibiotiques sont entre autres, la sélection de bactéries résistantes aux antibiotiques.
Le Tchad est un pays d'élevage par excellence et les produits de l'aviculture jouent un rôle nutritionnel, économique et socioculturel important et contribuent à la satisfaction des besoins en ouf et en viande de volaille (Mopate et Idriss, 2002).

Les maladies infectieuses et toxiinfections transmises par les aliments constituent un problème de santé publique et économique important au Tchad. Au cours des dernières décennies, les infections par les Salmonella ont été reconnues comme une menace pour la santé publique dans la plupart des pays en développement dont le Tchad (Karraouan, 2010). Des études menées dans d'autres pays comme le Burkina Faso, ont montré que $37 \%$ (Kagambega et al., 2011) des carcasses de poulets étaient contaminés par des salmonelles. L'accroissement et l'accumulation des résistances aux antibiotiques sont un autre aspect du problème de santé publique des salmonelloses (Courvalin, 2008). De nos jours, il est démontré qu'une partie des souches de Salmonella multirésistantes aux antibiotiques retrouvés chez l'homme sont d'origine animale et ont acquis leurs gènes de résistance en élevage avant d'être transmise à l'homme à travers les aliments (Fracois, 2008). L'infection est d'ailleurs très communément associée à la consommation de viande et de produits carnés, surtout ceux à base de volaille (Kimura et al., 2004). L'antibiorésistance conduit à l'utilisation de médicaments plus coûteux, ceci augmentant les coûts de soins de santé (OMS, 2005). En 
somme, la viande de poulet de chair commercialisée, est une source potentielle de souches de salmonelles résistantes aux antibiotiques pouvant être transmises à l'Homme; ce qui pose un problème de Santé publique.

Afin de protéger la santé du consommateur, il est nécessaire de prendre des mesures à toutes les étapes de la chaîne de production du poulet de chair. Au Tchad peu de données sont disponibles sur l'antibiorésistance liés à la consommation des aliments d'origines animales. La présente étude a été entreprise pour combler cette insuffisance. Dans l'optique d'évaluer l'impact de l'utilisation des antibiotiques sur la sensibilité des bactéries pathogènes de poules, cette étude a pour but de détecter les souches de Salmonella spp. non typhiques à partir des poulets de chair de Tchad et d'établir le profil de sensibilité aux antibiotiques des souches isolées.

\section{MATERIEL ET METHODES}

La démarche a combiné, d'une part, des investigations par enquête, et d'autre part des prélèvements et analyses en laboratoire. Les types et quantités d'antibiotiques utilisés en élevage ont été recensés, puis le profil de sensibilité aux antibiotiques des souches de salmonella isolées de poules déterminé.

\section{Enquête}

Une enquête concernant sept (7) sites d'élevages a été menée sur le circuit d'approvisionnement en aliments et intrants vétérinaires (médicaments et pré-mélanges); ainsi que, la conduite d'élevage de la volaille tels : l'application des règles sanitaires et la prophylaxie médicamenteuse.

\section{Echantillonnage}

La collecte des carcasses de poulet s'est effectuée dans 7 sites d'élevages et 5 marchés de la ville de N'Djaména. Il s'agit des sites de Guilmeye, Hillé Djidad, Farcha, Coq ville, Boutalwadi, Lamadji et Klessoum. Les marchés concernaient ceux du grand marché, du marché à Mil, du marché Al-afia, de Champs de Fil et du Dembé. Un questionnaire préétabli a permis de recueillir les informations nécessaires sur les sites concernés. L'échantillonnage a été fait de manière aléatoire en raison de cinq (5) carcasses de poulet par point de prélèvements. $\mathrm{Au}$ total 60 carcasses de poulets ont été collectées (par achat) dont 35 au niveau des sites d'élevages et 25 dans les points de vente. Les échantillons ont été prélevés individuellement et mis dans des sachets stomachers, puis conditionnés à $+4{ }^{\circ} \mathrm{C}$ dans une glacière pour le transport au laboratoire.

\section{Isolement, caractérisation préliminaire et identification des bactéries pathogènes}

La recherche des Salmonelles s'est réalisée selon la norme Française ISO 6579, en 4 étapes : le pré enrichissement, l'enrichissement, l'isolement et l'identification biochimique. Les parties telles : muscles et peau du coup ont fait l'objet d'une analyse bactériologique pour la recherche de salmonelles, en vue de déterminer le taux de portage.

\section{Pré-enrichissement}

Sur chaque carcasse de poulet, $25 \mathrm{~g}$ de peau de cou, et $25 \mathrm{~g}$ de muscle ont été prélevés. Les $25 \mathrm{~g}$ de muscle ont été prélevés après cautérisation de surface et prélèvement en profondeur. Chaque prélèvement a été suspendu dans un flacon stérile contenant 225 $\mathrm{ml}$ d'eau peptonée tamponnée, et incubé à 37 ${ }^{\circ} \mathrm{C}$ pendant $18 \mathrm{~h}$.

\section{Enrichissement}

Les prélèvements ont été pré enrichis à l'eau peptonée tamponnée. L'enrichissement été réalisé simultanément sur deux milieux. A l'aide de pipettes Pasteur, $0,1 \mathrm{ml}$ et $1 \mathrm{ml} \mathrm{du}$ pré enrichissement ont été prélevés et ajoutés respectivement à $10 \mathrm{ml}$ de Rappaport Vassilliadis Soja (RVS), puis à 10 $\mathrm{ml} \mathrm{du}$ bouillon Mueller Kauffman Tétrathionate novobiocine (MKTTn). Les tubes de MKTTn ont été incubés à $37{ }^{\circ} \mathrm{C}$ 
pendant 18 à 24 heures et les tubes de RVS à $42^{\circ} \mathrm{C}$, pendant $18 \mathrm{~h}$ à 24 heures.

\section{Isolement et caractérisation}

Les colonies suspectées Salmonella spp. ont subi des tests caractéristiques principaux, le test de catalase et la coloration de Gram et l'examen à l'état frais. Les isolats caractéristiques des salmonelles conservés à $20{ }^{\circ} \mathrm{C}$ dans le $\mathrm{BCC}$ additionné à $25 \%$ pour le test galerie Api $20 \mathrm{E}$.

\section{Identification sur galerie API 20 E}

Les bactéries de forme bâtonnet, catalase positif et Gram négatif ont été identifiées à l'aide des galeries API 20 E (BioMérieux, France) pour entérobactéries. La préparation de la galerie et de l'inoculum s'est opérée suivant les instructions du fabricant. Après incubation à $37^{\circ} \mathrm{C}$ pendant 24 heures, les résultats ont été analysés à l'aide du logiciel d'identification APIWEB.

\section{Tests de sensibilité aux antibiotiques}

L'antibiogramme a été réalisé par la méthode de diffusion sur gélose MuellerHinton de disques d'antibiotiques (Bio- Rad). L'interprétation des résultats a été faite selon les règles et les recommandations du Comité d'antibiogramme de la Société française de microbiologie (CA-SFM, 2011).

Après une culture bactérienne de 18 à $24 \mathrm{~h}$ en bouillon Trypticase-soja à $37^{\circ} \mathrm{C}$, des géloses Mueller Hinton sont inondées par les suspensions bactériennes, en diluant l'inoculum au 1/1000, pour obtenir l'équivalent de $10^{6} \mathrm{CFU} / \mathrm{ml}$. La lecture s'effectue après 18 à $20 \mathrm{~h}$ d'incubation à 37 ${ }^{\circ} \mathrm{C}$.

Les mesures des diamètres des zones d'inhibition ont été réalisées, puis les résultats interprétés selon les critères du comité de l'antibiogramme de la société française de microbiologie (CA-SFM). Les antibiotiques suivants ont été testés : Amoxicilline/acide clavulanique (AUG), Ciprofloxacine (CIP), Céfotaxime (CTX), Tétracyclines (TE), Triméthoprime-Sulfaméthoxazole (SXT),
Céftriaxone (CRO), Gentamycine (CN), Ampicilline (AMP), Chloramphénicol (C), Erythromycine (E), Imipenème (IMI), Acide fusidique (FC) et Céfoxitine (FOX).

\section{Analyse des données}

Pour l'analyse statistique des données, le logiciel XLSTAT 7.5.2 a été utilisé. L'analyse de variances (ANOVA) a été réalisée pour comparer les valeurs moyennes des différentes variables en utilisant les tests de Fisher au seuil de probabilité $\mathrm{p}=5 \%$.

\section{RESULTATS \\ Données des enquêtes}

Les résultats de l'application des règles sanitaires et de la prophylaxie médicamenteuse ont été présentés dans les Tableaux 1 et 2. Les informations obtenues à partir de la fiche d'enquête, ont permis de caractériser les sites d'élevage où ont été collectés les prélèvements. Il ressort de ces tableaux que parmi les activités, principalement les mesures d'isolement et le système de vaccination manquaient fortement aux règles de bonnes pratiques d'élevage.

\section{Prévalence de contamination par Salmonella spp. non typhique}

La prévalence de contamination par les salmonelles dans les échantillons de poulet de chair collectés au niveau des sites d'élevages et marchés de la ville de N'Djaména était de $68,33 \%(41 / 60)$. Les résultats mentionnés dans le Tableau 3, montre ainsi que parmi les 41 souches de Salmonelles non typhiques détectées ; des taux d'isolement de $34,15 \%$ et de $65,85 \%$ ont été obtenus respectivement sur les sites d'élevage et de point de vente. Les résultats ont montré que la prévalence des salmonelles variait significativement selon le site de prélèvement $(\mathrm{p}<0,05)$. Le degré de contamination des carcasses de poulets par Salmonella spp. varie en fonction de l'origine et a montré que les sites d'élevage sont les plus exposés.

Le Tableau 4 présente les résultats des analyses faites à partir des prélèvements sur la 
peau et au niveau des muscles de poulets de chair. La contamination des carcasses par Salmonella spp. varie significativement $(p<0,05)$ en fonction de la partie prélevée. Des taux respectifs de contamination de $26,66 \%$ et $41,66 \%$ sur les prélèvements sur les muscles et la peau.

\section{Susceptibilité des souches de Salmonella spp. isolées aux antibiotiques}

La résistance des souches a été testée vis-à-vis de 13 antibiotiques et les proportions d'isolats résistants sont illustrées dans le Tableau 5. Les souches de Salmonella spp. isolées ont montré une totale résistance $(100 \%)$ à l'Ampicilline, l'Erythromycine et à l'Imipenème.

De forts taux respectifs $87,80 \%$, $51,22 \%, 41,46 \%$ ont été observés avec l'Acide fusidique, la Tétracycline, la TriméthoprimeSulfaméthoxazole. Cependant, de fortes sensibilités ont été observées avec
Ciprofloxacine (100\%), au Céftriaxone $(92,68 \%)$ et à la Gentamicine $(95,12 \%)$. Par ailleurs l'étude a montré une résistance de salmonelles à l'ampicilline et imipenème $(100 \%)$.

\section{Multi-résistance des souches isolées aux antibiotiques}

La multi-résistance des souches de Salmonella spp. isolées dans cette étude varie de 3 à 7 antibiotiques. L'analyse de la multirésistance a permis d'obtenir les résultats mentionnés dans le tableau 5. La recherche à la multirésistance a montré que : 29,27\%, $17,07 \%$ et $14,63 \%$ des souches ont été résistantes respectivement à $46 \%, 53,85 \%$ et $38,46 \%$ des antibiotiques testés. La résistance aux quatre antibiotiques (Ampicilline, Erythromycine, Imipenème et Acide fusidique) a été la plus observée avec un pourcentage de $34,15 \%$ des souches.

Tableau 1: Application des règles sanitaires dans les sites d'élevage visités.

\begin{tabular}{lc}
\hline Prophylaxie sanitaire & Fréquence de non respect des règles (\%) \\
\hline Vide sanitaire & $57,14(\mathrm{n}=4)$ \\
\hline Elevage en bande unique & $57,14(\mathrm{n}=4)$ \\
\hline Mesures d'isolement & $85,71(\mathrm{n}=6)$ \\
\hline Désinfestation & $57,14(\mathrm{n}=4)$ \\
\hline Hygiène des locaux et aliments & $71,42(\mathrm{n}=4)$ \\
\hline
\end{tabular}

Tableau 2 : Fréquence d'utilisation médicamenteuse et vaccination dans les sites d'élevages visités.

\section{Prophylaxie médicamenteuse}

Fréquence (\%) de non respect des règles

\begin{tabular}{ll}
\hline Utilisation antibiotiques & $57,15(\mathrm{n}=4)$ \\
\hline Vaccination & $71,43(\mathrm{n}=3)$
\end{tabular}


Tableau 3: Répartition des souches de Salmonella spp. détectées en fonction des sites de collecte.

\begin{tabular}{lccc}
\hline Origine & Nombre d'échantillons & Salmonella spp. & p-value \\
\hline Site d'élevage & 35 & $65,85 \%(\mathrm{n}=27)$ & \multirow{2}{*}{$p<0,05$} \\
\cline { 1 - 3 } Point de vente & 25 & $34,15 \%(\mathrm{n}=14)$ & \\
\cline { 1 - 3 } Total & 60 & $68,33 \%(\mathrm{n}=41)$ & \\
\hline
\end{tabular}

Tableau 4: Répartition des souches de Salmonella spp. en fonction de la partie prélevée sur les carcasses de poulets de chair.

\begin{tabular}{lccc}
\hline Partie prélevée & Nombre d'échantillons & Salmonella spp. & p-value \\
\cline { 1 - 3 } Muscle & 60 & $16(26,66 \%)$ & \\
\cline { 1 - 3 } Peau de cou & 60 & $25(41,66 \%)$ & $p<0,05$ \\
\cline { 1 - 3 } Total & 120 & 41 & \\
\hline
\end{tabular}

Tableau 5: Susceptibilité aux antibiotiques des souches de Salmonella spp. isolées.

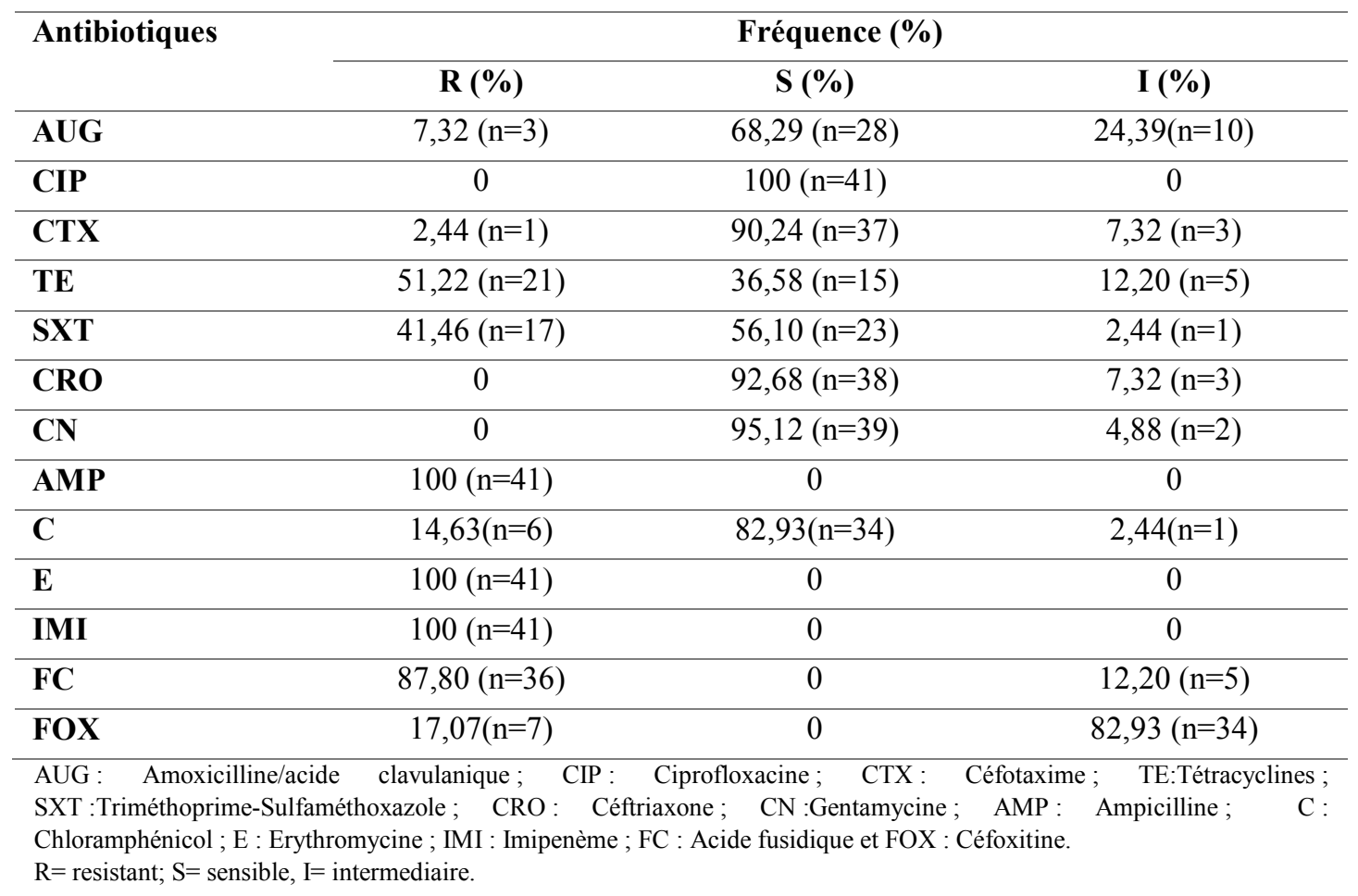


Tableau 6 : Multi-résistance des souches de Salmonella spp. aux antibiotiques isolées dans les sites d'élevages et marchés visités.

\begin{tabular}{lcc}
\hline Antibiotiques & Nombre de souche & Pourcentage de résistance (\%) \\
\hline $3 \mathrm{Ab}$ & 2 & 4,89 \\
\hline $4 \mathrm{Ab}$ & 14 & 34,15 \\
\hline $5 \mathrm{Ab}$ & 6 & 14,63 \\
\hline $6 \mathrm{Ab}$ & 12 & 29,27 \\
\hline $7 \mathrm{Ab}$ & 7 & 17,07 \\
\hline $\mathrm{TOTAL}$ & 41 & 100 \\
\hline \multicolumn{1}{r}{$\mathrm{Ab}=$ Antibiotiques } & &
\end{tabular}

\section{DISCUSSION}

\section{Données des enquêtes}

Les résultats de l'enquête ont montré qu'en moyenne plus de la moitié $(50 \%)$ des sites d'élevage ne possédant pas un plan sanitaire bien défini et $85,71 \%$; et ne possèdent pas de mesures d'isolement essentielles pour réduirent les risques sanitaires.

Les enquêtes révèlent que $71,43 \%$ des sites d'élevages ne procèdent pas la vaccination de leurs volailles et $57,15 \%$ utilisent de façon arbitraire les antibiotiques pour le traitement avicole. Ces résultats sont en accord avec ceux de Nana (2000) qui a montré que la plupart des éleveurs n'appliquent pas les bonnes règles d'élevage.

\section{Prévalence de contamination par Salmonella spp. non typhique}

Le degré de contamination des carcasses de poulets par Salmonella spp. varie en fonction de l'origine et a montré que les sites d'élevage sont les plus exposés $(65,85 \%)$. Cette prévalence est largement supérieure aux résultats obtenus par Karraouan et al. (2010) (20,31\%) au Maroc ; Kagambega et al. (2011) (37\%) au Burkina Faso ; Alloui et al. (2013) (50\%) en Algérie ; Ezzat et al. (2014) (18,5\%) en Egypte. Par ailleurs nos résultats se rapprochent de ceux rapportés au Sénégal par Fofana (2004) $(62,5 \%)$ et au Cameroun par Ngoune et al. (2009) (67\%) et en Côte d'Ivoire par Bonny et al. (2011) $(61,87 \%)$ sur des échantillons de poulets de chair. Comparée aux autres types d'oiseaux (Ploceus cucullatus), la prévalence de Salmonella spp. obtenue par Piebeng et al. (2014) $(78,46 \%)$ est supérieure à celle de notre étude.

Le taux relativement élevé de la prévalence pourrait être expliqué par plusieurs facteurs tels: la taille de l'échantillon qui n'est pas représentative de l'ensemble des sites de N'Djamena, le nonrespect des conditions d'hygiène, l'absence des programmes de surveillance sanitaire et l'utilisation prophylactique d'antibiotiques non conforme contre les salmonelles. Les sites étaient plus contaminés que les marchés, dus peut-être à l'encombrement des déchets (fientes et ordures) et l'insalubrité de l'environnement et des locaux d'élevages. Ce constat corrobore avec les résultats de Cardinale et al. (2004) qui ont montré qu'au Sénégal : $28,6 \%$ des fientes issues de 70 fermes de poulets de chair sont infectées par Salmonella spp. La peau du cou était plus contaminée. Ce constat est en accord avec les travaux de Fofana (2004) et Diouf (2006) au Sénégal ; qui avaient trouvé des pourcentages de contamination respectifs de $77,5 \%$ et $29 \%$ sur les prélèvements respectivement de la peau et contre $32,5 \%$ et $19 \%$ au niveau des muscles. Cette prévalence serait due au fait que la peau est permanemment en contact avec l'environnement extérieur.

\section{Susceptibilité des souches de Salmonella spp. isolées aux antibiotiques}

Les souches de Salmonella spp. isolées ont montré une totale résistance $(100 \%)$ à l'Ampicilline, l'Erythromycine et à l'Imipenème. L'ampicilline appartient à la 
famille des bêtalactamines qui agissent en inhibant la synthèse du peptidoglycane après fixation sur un récepteur protéique membranaire : le PBP (Penicilllin Binding Protein) (Armengaud et al., 1994 ; Yala et al., 2001). Les souches étudiées résistent à ces antibiotiques par la production de bêtalactamases qui les inactivent antibiotiques (Armengaud et al., 1994).

Courvalin (2008) a montré que les antibiotiques qui appartiennent à la même classe agissent par le même mécanisme d'action et que les bactéries cibles peuvent leur résister par un mécanisme identique. De taux respectifs $87,80 \%, 51,22 \%, 41,46 \%$ ont été observés avec l'Acide fusidique, la Tétracycline, la TriméthoprimeSulfaméthoxazole. Ils se rapprochent de ceux trouvés par Ngoune (2009) en ce qui concerne les Tétracyclines (45\%) et le Triméthoprimesulfaméthoxazole $(40,14 \%)$.

Par contre, elles sont inférieures à celles obtenues par Diouf (2006) qui sont de $73,24 \%$ pour les tétracyclines et de $64,78 \%$ pour la Triméthoprime-sulfaméthoxazole. Les tétracyclines, sont d'anciennes molécules largement utilisées en première intention. La résistance à ces molécules est assez connue et serait généralement due à un gène plasmidique qui peut être acquis assez facilement par les bactéries. Par ailleurs, c'est un antibiotique bactériostatique à large spectre, actif contre les bactéries Gram+ et les Gram- (Armengaud et al., 1994). Cependant, de fortes sensibilités ont été observées avec Ciprofloxacine (100\%), au Céftriaxone $(92,68 \%)$ et à la Gentamicine $(95,12 \%)$. L'enquête a montré que les fluoroquinolones ne sont très peu ou pas utilisés dans la filière avicole au Tchad ; ce qui pourrait justifier cette sensibilité des souches. L'antibioresistance des souches isolées au chloramphénicol; a montré un taux inférieur aux résultats de Somda (2012) au Burkina Faso et Bonny et al. (2011) de la Côte d'Ivoire, qui avaient trouvé respectivement $85 \%$ et $41,7 \%$.

Par ailleurs l'étude a montré une résistance de salmonelles à l'ampicilline et imipenème $(100 \%)$, similaire à celle de Wahome et al. (2014) au Kenya (100\% à l'ampicilline) supérieure à celle obtenue par Compaore (2006) au Burkina Faso qui était $90 \%$. Certains auteurs tels Mzungu et al. (2016) au Nigéria, ont trouvé des profils de résistances différentes sur leurs souches testées : $\quad 100 \%$ à l'Ampicilline et l'Amoxicilline; $90,9 \%$ à Amoxicilline-Acide Clavulanique; 45,5\% à l'Acide Nalidixique. Pour ce qui concerne la sensibilité des souches $100 \%$ étaient sensibles à la Gentamicine; $90,9 \%$ à la Ciprofloxacine et au Cefotaxime.

\section{Multi-résistance des souches isolées aux antibiotiques}

La multi-résistance obtenue dans cette étude est comparable à celle d'autres auteurs. Mzungu et al. (2016) au Nigéria, ont trouvé des profils de résistances différentes sur leurs souches testées : $100 \%$ à l'Ampicilline et l'Amoxicilline; $90,9 \%$ à Amoxicilline-Acide Clavulanique; $45,5 \%$ à l'Acide Nalidixique. Pour ce qui concerne la sensibilité des souches $100 \%$ étaient sensibles à la Gentamicine; 90,9\% à la Ciprofloxacine et au Cefotaxime. $\mathrm{Au}$ Ghana, les souches isolées de poulets par Brown et Alhassan (2014) étaient résistantes à l'Ampicilline, l'Erythromycine et à la Tétracycline. Cette multi-résistance peut s'expliquer d'une part du fait que les bactéries résistantes aux bétalactamines produisent des bétalactamases qui inactivent les antibiotiques appartenant à cette famille (Armengaud et al., 1994). D'autre part la présence de résistances croisées causées par les antibiotiques appartenant à la même classe. En effet, les antibiotiques qui appartiennent à la même classe agissent par le même mécanisme d'action (Courvalin, 2008).

Les antibiotiques, étant utilisés de façon désordonnée dans les fermes à Djamena, créent inévitablement une pression de sélection qui favorise le développement des bactéries multirésistantes. Cette multirésistance est beaucoup plus inquiétante, car ce sont des molécules d'antibiotiques utilisées couramment lors du traitement des salmonelloses humaines au Tchad. Ce même constat a été observé par Ngoune et al. (2009) au Cameroun. 


\section{Conclusion}

$\mathrm{Au}$ terme cette étude qui visait à montrer l'impact de l'utilisation des antibiotiques sur la sensibilité des bactéries pathogènes de poules des fermes de la ville de Djaména. L'étude a montré une multirésistance des souches de Salmonella isolées ; à l'Ampicilline, à l'Erythromycine, à l'Imipenème, à l'Acide fusidique, aux Tétracyclines.

L'importance de la résistance bactérienne observée dans cette étude reflète l'usage antérieur des antibiotiques dans les élevages à titre curatif ou prophylactique. Il serait donc nécessaire de mettre en place une bonne pratique d'hygiène avicole et de procéder à une cuisson parfaite des aliments, afin d'éviter toute contamination.

\section{CONFLIT D'INTERETS}

conflit d'intérêts.

Les auteurs déclarent qu'il n'ya pas de

\section{Contribution des auteurs}

Conception du projet d'article réalisé par MKS ; Conduite des activités de recherche et collecte des données réalisées par HA Traitement des données collectées réalisé par HA ; Rédaction de l'article réalisé par Marius KS ; Mise en œuvre du projet et aide au financement réalisé par BBA ; NB a participé à la rédaction de l'article; Lecture et correction de l'article réalisé par AST.

\section{Remerciements}

Nous remercions, le Laboratoire de Biologie de la Faculté des Sciences Exactes et Appliquées de L'Université de N'Djaména (Tchad), le Centre de Recherche en Sciences Biologiques, Alimentaires et Nutritionnelles (CRSBAN) pour leur appui dans la conduite des travaux de recherche.

\section{REFERENCES}

Alloui N, Guergueb N, Ayachi A. 2013. Relation entre les pratiques d'hygiène d'abattage et la contamination bactérienne des carcasses de poulets dans la région de Biskra (Algérie). Conference: Recherche Avicole et Palmipèdes à Foie Gras. La Rochelle : 1-
5.

https://www.researchgate.net/publication $/ 237010462$

Armengaud M, Astruc J, Aubertin J, Auvergnat JC, Beaucaire G, BecqGiraudon B, Bertrand JL. 1994. Antibiotiques: Les Maladies Infectieuses. APPIT (ed) 2M2: France; p.671.

Bada-Alambedji R, Cardinal E, Biagui C, Akakpo AJ. 2004. Recherche de résidus de substances à activité antibactérienne dans la chair de poulet consommée dans la région de Dakar (Sénégal). Bull. Acad. Vét., 157(2): 6770. www.academie-veterinaire-france.fr

Biagui C. 2002. Utilisation des médicaments vétérinaires en élevage avicole dans la région de Dakar; qualité de la viande à travers la recherche de résidus de substances à activité antimicrobienne (Antibiotiques). Thèse de Doctorat en Médecine Vétérinaire, Ecole Inter-Etats des Sciences et Médecine Vétérinaires, Université Cheick Anta Diop de Dakar, p. 153. http://www.beep.ird.fr/collect/eismv/inde x/assoc/TD02-8.dir/TD02-8.pdf

Bonny AC, Karou TG, Atobla K, Bohoua LG, Niamkey LS. 2011. Portage de Salmonella au niveau du gésier cru de poulets exposés a la vente à Abidjan, Côte d'Ivoire. J. Appl. Biosci., 47: 32303234.

http://www.m.elewa.org/JABS/2011/47/ 5.pdf

Brown C, Alhassan AN. 2014. Multipleantibiotic-resistant bacteria from cockroaches trapped from a public hospital and a nearby students' hostel in Accra, Ghana. Int. J. Biol. Chem. Sci., 8(4): 1859-1864. http://ajol.info/index.php/ijbcs

Cardinale E, Colbachini P, Perrier-GrosClaude JD, Gassama A, Aidara-Kane A. 2001. Dual emergence in food and human of a novel multiresistant serotype of Salmonella in Senegal: Salmonella enterica subsp. enterica serotype 35:c:1, 2. J. Clin. Microbiol., 39(6): 2373-2374. 
CA-SFM 2011. Comité de l'antibiogramme de la société française de microbiologie : Recommandations 2011. Ed de janvier 2011. http://www.sfm.asso.fr/.

Compaore L. 2006. Analyse phénotypique et génotypique de la résistance de sept espèces d'Entérobactéries aux antibiotiques. Thèse Pharmacie Université de Ouagadougou, p.110.

Courvalin P. 2008. La résistance des bactéries aux antibiotiques: combinaison des mécanismes biochimiques et génétiques. Bull. Acad. Vét., 161:7-12. $\mathrm{http} / / /$ academieveterinaire.free.fr/bulletin /pdf/2008/numero01/07.pdf

Diouf KCN. 2006. Surveillance de la résistance aux antibiotiques des souches de Salmonella spp. et Escherichia coli isolées de la viande de poulet de chair au Sénégal. Mémoire de DEA, EISMV, Dakar, p.60. http://www.beep.ird.fr/collect/eismv/inde x/assoc/MEM06-6.dir/MEM06-6.pdf

Ezzat ME, Shabana I I, Esawy AM, Elsotohy ME. 2014. Detection of virulence genes in Salmonella serovars isolated from broilers. Animal and Veterinary Sciences, 2(6): 189-193. DOI: 10.11648/j.avs.20140206.16.

Fofana A. 2004. Résistance aux antibiotiques des souches de Salmonella spp. et Escherichia coli isolées de la viande de poulets de chair au Sénégal. Mémoire de DEA, EISMV, Dakar, p.43. http://www.beep.ird.fr/collect/eismv/inde x/assoc/MEM06-6.dir/MEM06-6.pdf

François X. 2008. Salmonelles non typhiques d'origine animale et résistance aux antibiotiques. Bull. Acad. Vét., 163(3): 221-234. www.academie-veterinairedefrance.org/

Gaudel P. 2013. Juste usage des antibiotiques à l'hôpital de Brabois adultes CHU de Nancy. Bilan du rôle du pharmacien et de l'équipe opérationnelle en infectiologie de 2006 à 2012. Thèse de Doctorat Université de Lauraine, p.120. http://docnum.univlorraine.fr/public/BUPHA_T_2013_GA UDEL_PAULINE.pdf
Humphrey T. 2000. Public-health aspects of Salmonella infection. In Salmonella in Domestic Animals, Way C, Way A (eds). Oxon: CABI Publishing: 245-263. doi.org/10.1079/9780851992617.0245.

International Standard Organization (ISO 6579) 2002. Microbiologie des aliments. Méthode horizontale pour la recherche des Salmonella spp. Saint-Denis, p.27. www.iso.org/iso/catalogue_detail.htm?cs number $=29315$

Kagambega A, Haukka K, Sitonen A, Traore A S, Barro N. 2011. Prevalence of Salmonella enterica and the hygiene indicator. Escherichia coli in raw meat at markets in Ouagadougou, Burkina Faso. J. Food Prot., 74: 1547-1551. Doi: 10.1089/fpd.2011.1071

Karraouan B A, Fassouane H, El-Ossmani NCO, Charafeddine BB. 2010. Prévalence et gènes de virulence des Salmonella isolées des viandes hachées crues de dinde à Casablanca (Maroc). Revue Méd. Vét., 161(3): 127-132. http://www.revmedvet.com/2010/RMV1 61_127_132.pdf.

Khan AJ, Ramswaroop R, Shaheen K, Iqbal A. 2013. Prevalence, characterization and detection of Salmonella spp. from various meat sources. Ad. An. Vet. Sci., 1(1S): $\quad 4-8$ http://www.nexusacademicpublishers.co $\mathrm{m} /$ journal $/ 4$

Kimura AC, Reddy V, Marcus R, Cieslak PR, Moehle-Boetani JC, Kassenborg HD, Segler SD, Hardnett FP, Barrett T, Swerdlow DL. 2004. Chicken consumption is a newly identified risk factor for sporadic Salmonella enterica serotype Enteritidis infections in the United States: a case-control study in Food Net sites. Clin. Infect. Dis., 38(S3): 244-252. DOI:10.1086/381576

Kingsley RA, Msefula CL, Thomson NR, Kariuki S, Holt KE, Gordon MA, Harris D, Clarke L, Whitehead S, Sangal V, Marsh K, Achtman M, Molyneux ME, Cormican M, Parkhill J, MacLennan CA, Heyderman RS, Dougan G. 2009. Epidemic multiple drug resistant Salmonella Typhimurium causing 
invasive disease in sub-Saharan Africa have a distinct genotype. Genome Res., 19: 2279-2287.

DOI:10.1101/gr.091017.109

Mzungu I, Inabo HI, Olonitola SO, Aminu M. 2016. Antibiotic susceptibilities of Salmonella species prevalent among children of 0-5 years with diarrhea in Katsina state, Nigeria. Arch. Med. Biomed. Res., 3(1): 39-51. www.ajol.info/index.php/ambr/article/do wnload/133012/122635

Molla B, Mesfin A. 2003. A survey of Salmonella contamination in chicken carcass and giblets in central Ethiopia. Rev. Med. Med. Vet., 154(4): 267-270.

www.revmedvet.com/2003/RMV154_26 7 270.pdf

Mopate LY, Idriss OA. 2002. Etat de l'aviculture familiale au Tchad et les perspectives de son développement. Etudes et Recherches Sahéliennes, Insah. Bamako, 6(7): 7-15. http://rsttchad.org/index.php/annuaire-deschercheurs-et-enseignants-ercheurs/238mopate-logtene-y

Nana GS. 2000. Les points à risque de la contamination microbiologique de la viande de poulet de chair dans la région de Dakar. Thèse Médecine Vétérinaire, EISMV, Dakar, 108p.

www.beep.ird.fr/collect/eismv/index/ass oc/TD00-8.dir/TD00-8.pdf

Ngoune LT, TANEDJEU SK, MBOFUNG CMF. 2009. Impact de l'utilisation des antibiotiques sur la sensibilité des bactéries pathogènes de poules dans la ville de Ngaoundéré. Camer. J. Experiment. Biol., 5(2): 52-61. http://www.ajol.info/browsejournals.php

OMS. 2005. Salmonelles résistantes aux antimicrobiens.

http://www.who.int/foodsafety/fs_manag ement/No_03_Salmonella_Apr05_fr.pdf
Piebeng GNK, Tamungang SA, Fusingwa C, Ndukum JA, Bayoï JR, Gapessie L, Katte B. 2014. Profils bactériens et fongiques dans les fèces des tisserins villageois (Ploceus cucullatus) dans la ville de Dschang et ses environs (OuestCameroun). Int. J. Biol. Chem. Sci., 8(4): 1771-1783. http://ajol.info/index.php/ijbcs

Somda N. 2012. Sérotypage et antibiorésistance des souches de Salmonella spp. et de Shigella spp. isolées chez les enfants de moins de cinq ans en milieux urbain et rural au Burkina Faso. Mémoire DEA, Université de Ouagadougou, p.60.

Voetsch AC, Van Gilder TJ, Angulo FJ, Farley MM, Shallow S, Marcus R, Cieslak PR, Deneen VC, Tauxe RV, Emerging Infections Program FoodNet Working Group. 2004. Food-Net estimate of the burden of illness caused by non-typhoidal Salmonella infections in the United States. Clin. Infect. Dis., 38(S3): 127-134.

Wahome CN, Okemo PO, Nyamache AK. 2014. Microbial quality and antibiotic resistant bacterial pathogens isolated from groundwater used by residents of Ongata Rongai, Kajiado North County, Kenya. Int. J. Biol. Chem. Sci., 8(1): 134-143. http://ajol.info/index.php/ijbcs.

White PL, Baker AR, James WO. 1997. Strategies to control Salmonella and Campylobacter in raw poultry products. Rev. Sci. Tech., 16: 525-541. PMID:9501366

Yala D, Merad AS, Mohamedi D, OuarKorich MN. 2001. Classification et mode d'action des antibiotiques. Médecine $d u$ Maghreb, 21: p.12. http://www.slideserve.fr/classificationet-mode-d-action-des-antibiotiques. 